\title{
Towards Web 3.0: An Application Oriented Approach
}

\author{
*Dr V.Kiran Kumar, Asst Prof \\ Department of Computer Science, Dravidian University
}

\begin{abstract}
The World Wide Web (WWW) is global information medium, where users can read and write using computers over internet. Web is one of the services available on internet. The Web was created in 1989 by Sir Tim Berners-Lee. Since then a great refinement has done in the web usage and development of its applications. In this paper we would like to present different stages of web growth starting from its inception to the present web in terms of its technologies and applications.
\end{abstract}

\section{Introduction}

The dream behind creating the web was to create a common information space in which people communicate by sharing information ${ }^{1}$. The World Wide Web (WWW) is a collection electronic document over internet. Each electronic document is called as a webpage and it can contain text, image, video and audio etc., and the collection of such pages can be called as a Website. The WWW can be viewed as huge client-server system, where millions of servers are distributed over internet containing electronic documents. A server accepts requests from clients and transfers files / web pages accordingly to client.

The web was created in 1989 by Sir Tim Berners-Lee, working at CERN in Geneva, Switzerland ${ }^{1}$. Since then, Berners-Lee has played an active role in guiding the development of web standards starting from Web 1.0 to the present Web 3.0, which we can call it as an intelligent web or Semantic web.

The rest of the paper is organized as follows: Section 2 describes about the related works happened so far. Section 3 and Section 4 discusses about the technologies and applications of Web 1.0 and Web 2.0. Section 5 focuses on the Web 3.0, which aims to develop intelligent agents and Section 6 ends with conclusion.

\section{First Generation Web (Web 1.0)}

The first implementation of web is known as informational web, which was developed from 1991 onwards. Initially they were focused on companies in preparing broachers or catalogs for marketing purpose. This web can also be called as "Read-only web", because information is created by small number of authors targeting for very large number of users. The users have a limited role of reading information from web with small interaction using hyperlinks, hence these web pages are comes under static web pages. Static Web page contains information which does not update frequently by the owner.

The first shopping carts applications, which does e-commerce on web was developed in this era. Their goal is to prepare broachers or catalogs to present products to customers for marketing purposes. "Craigslist is a classified advertisements website with sections devoted to jobs, housing, personals, for sale, items wanted, services, community, gigs, resumes, and discussion forums"2. In this era, companies develop application for users to use, but the users can't see how the application works and change it ${ }^{3}$. These web applications are not falls under open source category, where everybody can see the code and change it as per their requirements. The web sites developed in this era does not support AJAX (Automatic Java Script and XML) techniques, profiles, blogs and let alone option to comment $t^{4}$. The authors can develop web applications with some knowledge on HTML and some image editing skills. The most popular web sites in this era are

- Hotmail was introduced in 1995. This was the first free mailing facility provided to contact people through e-mail ids. A person needs to register with hotmail to get an e-mail id. Once registered he can send or receive message through this site.

- Geocities was the most popular website to create our own free web pages. In 1997 Geocities was the fifth most popular website, with over 500,000 homepages created.

- The World Wide Web Worm (WWWW) is the first search engine developed in 1993. This engine uses titles and headers of web pages for indexing purpose. The most popular search engine Altavista was developed by Google in this era. This will index the web sites created by authors and returns as search results for the user.

- The first instant messenger program ICQ (I seek you) was created in 1996, where people can chat online. Chatting is a program which sends messages to others instantly online.

- The first audio from the web was transmitted in the Realplayer format. This was in a time of .wav files and slow 36k modems: not a good combination. Real had created the solution with their applications 


\section{Second Generation Web (Web 2.0)}

The lack of active interaction of users with the web leads to the next generation web i.e., Web 2.0. In Web 1.0, the web creators (or) authors are very few and role of users or clients just consume the existing web content. But in Web 2.0 any user can become an author (or) content creator and post his information to share with others on the web. The Web 1.0 is aims to connect the people where as Web 2.0 aims to connect communities; hence this web is called as Social Web or "Read-Write-Publish Web". It consists of number of online tools where people share their opinions, experiences and thoughts online. Web 2.0 was officially defined in 2004 by Dale Dougherty, vice-president of O'Reilly Media. When comparing with Web 1.0, there is a change in social aspect of connecting the people but technically there is no change. Because the data on the web still can't understand by the computer'.

A popular innovation in Web 2.0 is "mashups,". A mashup is a web page, or web application, that uses content from more than one source to create a single new service displayed in a single graphical interface ${ }^{7}$. For example, street addresses in the website can visualize in google maps in finding locations.

In 1999, the "Read-Write-Publish" era has started with contributions from LiveJournal (Launched in April, 1999) and Blogger (Launched in August, 1999). Some of the most popular and remarkable developments of Web 2.0 are Twitter, YouTube, eZineArticles, Flickr and Facebook, Wikis, RSS Feeds, Flash etc., each has its own advantage of sharing different types of content like images, videos, text etc., to share on the web. Some of the most popular applications are

- The term web-log, or blog, was coined by Jorn Barger in 1997 and refers to a simple webpage consisting of brief paragraphs of opinion, information, personal diary entries, or links, called posts, arranged chronologically with the most recent first, in the style of an online journal (Doctorow et al., 2002). The important aspect of blog is that a visitor can add his own comment in the blog entry. As the technology is developing, bloggers have begun to upload multimedia content into their blogs and they called now as photo-blogs, video-blogs(vblogs) and mobile-blogs (where bloggers can upload their data through mobile phones).

- A wiki3 is a webpage or set of webpages that can be easily edited by anyone who is allowed access (Ebersbach et al., 2006). These pages contain 'Edit' button to edit the content, add the content or even delete the content using online editing tool. Allowing access to anyone can sometimes lead to the malicious editing and vandalism (Stvilia et al., 2005). This can be restricted by allowing authorised users to edit the webpages.

- The most popular and fastest growth of web service is multimedia, which includes YouTube (Videos), Flickr (Photographs) etc., These services provides the users to publish their own multimedia content to share it on the web. Few example include http://www.flickr.com/, http://www.ourpictures.com/, http://www.youtube.com/, http://eyespot.com/

- Podcasts $^{6}$ (Coined by Ben Hammersley in a Guardian article on 12th February 2004:) are audio recordings, usually in MP3 format, of talks, interviews and lectures, which can be played either on a desktop computer or on a wide range of handheld MP3 devices. These files are usually in MP3 format and can upload it into server. The users who wish to listen these files can download and use. Example are http://btpodshow.com/, http://www.audblog.com/.

- As the web content is updating very frequently by the large number of users, it is difficult to visit each website for getting update content. To solve this problem we use RSS feeds to enable and include in the websites. These feeds get the regular update of web sites and store it into a user desktop through a software tool known as 'aggregator'. The uses need to subscribe concerned RSS feed for regular update ${ }^{8}$.

\section{Future Generation Web (Web 3.0)}

The Web grows at an astounding pace due to the number of authoring tools available for the users to create the web content instantly. The huge success of the web is due to the freedom it gives. But still the existing web content is designed for the human consumption and can't be processed by machines. For example, search engines helps us to retrieve the web content for us, but still the human intervention is needed to get the proper result. Hence, the next generation web focuses on creating web content for human consumption as well as machines to understand it to interpret. This future generation web can be called as Web 3.0 or Semantic Web.

According to the W3C, "The Semantic Web provides a common framework that allows data to be shared and reused across application, enterprise, and community boundaries. ${ }^{\prime 9}$ The term was coined by Tim Berners-Lee for a web of data that can be processed by machines ${ }^{10}$.

Earlier HTML metadata tags were used for providing machine understandable data for web applications. Metadata is data (information) about data. These tags reside in the $<$ HEAD $>$ section of the HTML file. $<$ META $>$ has two attributes, NAME and CONTENT, which can be used to store the metadata schema. The current search engines use these metadata tags to index the WebPages and Websites. Due to its very simplicity, 
these tags were not used for describing complex properties. In 2004, World Wide Web Consortium (W3C) has recommend Resource Description Framework (RDF) as a part of Semantic web activity. RDF is a general purpose language for representing information about the resources on the web. Further it is designed to support interoperability between applications that can exchange machine-understandable information on the web. The lack of expressiveness of RDF leads to introduction of Resource Description Framework Schema (RDFS) and ontology. Figure.1 represents different languages which are used in designing Semantic Web applications. The primary purpose of these languages is to represent machine-understandable information and to support interoperability between applications on web. Once we add semantics to the website, we can design semantic web applications for the users to use.

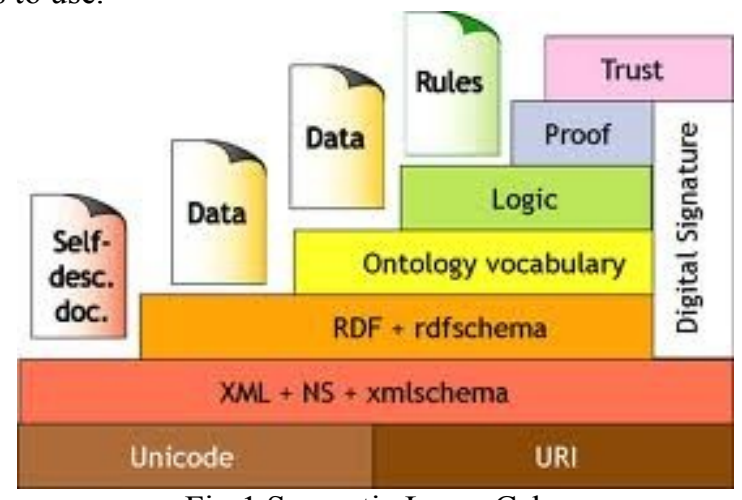

Fig.1 Semantic Layer Cake

An 'Agent' is semantic web application which roams around the web and searches the information as per the user preferences given. According to Tim Berners-Lee, software agents will be responsible for coordinating searches, comparing and negotiating on the web and greatly reducing the user effort (Berners-Lee et al.2001) ${ }^{11}$. For example, if a student wishes to join a particular course in some country, then he approaches a particular agent and given his preferences like course fee, faculty details, location of the institution etc., Then the agent go around the web with preferences given by the user and collects some of the choices and return to the user for his decision. These 'Agents' looks like a conventional software application, but there is a clear distinction exists like 'Agents' are semi-autonomous, pro-active, adaptive and long lived. There are different types 'Agents' which include Interactive Agents, Adaptive Agents, Mobile Agents, Coordinative Agents, Intelligent Agents and Wrapper Agents. Each 'Agent' has its own advantage of how to approach to other 'Agents' and environments. One example of such application is 'tripit' (https://www.tripit.com/), which is a personal software travel assistant that automatically organizes your entire travel plan.

The other type of application which uses metadata annotations, ontologies and semantic web protocols on desktop computers that allows the integration of desktop application and web is called 'Semantic Desktop' 12 . These applications allow us to semi-automate the information update process with the help of 'Agents'. The primary intention of Semantic Web is to enrich the existing WWW with data. This same technology is applying for personal computer to access information stored on it. Here all the files like photos, audio, video, e-mail, files etc., are used as web resources. These resources will be represented in RDF syntax and can be used by any application. A local semantic web server can be used to access this information. Once this representation is done, semantic search can be implementing to get better results and can also contact other desktops with the help of 'Agents'. Barreau and Nardi ${ }^{13}$ analyzed the searching behavior on desktop computers in 1995 and identified two different search strategies when users are looking for information that is stored on their desktop computers. The first strategy deals with 'path' and looks for the folders and directories for the document. The second strategy deals with 'full text search', which is used today in almost every personal computer. The Semantic Desktop ${ }^{14}$ is not one user interface or one form of information storage, but it provides common ground to integrate with other applications. The system is capable of answer natural language queries which give better results than the present search techniques available as discussed. $\mathrm{KDE}^{15}$ (K Desktop Environment) is an example application of Semantic Desktop. The implementation of the search capability within KDE 4.0 was based on RDF and RDFS. The key features of interest with these technologies included the extensible and adaptive data model, and fast storage and retrieval of data.

\section{Conclusion}

In this paper we would like to focus on applications designed since inception of the web. The first generation web focuses on how to connect people by designing web sites. The users can just read the web content only; the applications were designed with little bit dynamic content like submission of forms. The second generation web focuses on connecting the communities and provides access to the users to read the 
content as well as write content online. With the help of many authorized online tools available the users can read and write the content. Hence, applications are designed in such a way to focus social networking sites, multimedia sharing, mashup, blogs, wikis etc., The next generation web focuses on how to provide machineunderstandable format and how to consider the web as database by providing semantics to the web content. Then users can design applications on the web database for the humans to reduce the burden at great extent.

\section{References:}

[1]. World Wide Web Consortium (W3C). Introduction to Web Accessibility. Available at http://www.w3.org/WAI/intro/accessibility.php (Accessed on 04/01/2008)

[2]. http://en.wikipedia.org/wiki/Craigslist

[3]. http://computer.howstuffworks.com/web-10.htm

[4]. http://www.yvoschaap.com/weblog/rip_a tribute to web 10

[5]. "Key difference between Web 1.0 and Web 2.0", Graham Cormode and Balachander Krishnamurthy.

[6]. http://technology.guardian.co.uk/online/story/0,3605,1145689,00.html [last accessed 14/02/07].

[7]. Fichter Darlene, What Is a Mashup? http://books.infotoday.com/books/Engard/Engard-Sample-Chapter.pdf ( retrieved 12 august 2013)

[8]. Really Simple Syndication and Rich Site Summary. What is Web 2.0. Available at http://www.rss-specifications.com/what-is-web2.htm (Accessed on 04/01/2008)

[9]. "W3C Semantic Web Activity". World Wide Web Consortium (W3C). November 7, 2011. Retrieved November 26, 2011.

[10]. Berners-Lee, Tim; James Hendler and Ora Lassila (May 17, 2001). "The Semantic Web". Scientific American Magazine. Retrieved March 26, 2008.

[11]. Karin K.Breitman, Macro Antonio Casanova, Walter Truszkowski. Semantic Web - Concepts, Technologies and Applications

[12]. Breitman, K.K; Truszkowski; Felicissiomo (2006) The automatic semantic desktop; Helping users copy with information system and complexity. In Proceedings of IEEE International workshop,pp.156-162.

[13]. Barreau,D.,Nardi,B.A.:Finding and reminding: File organization from the desktop. (1995)

[14]. Stefan Deckerand Martin Frank.The social semantic desktop.In Proc. Of the WWW 2004 Workshop Application Design, Development and Implementation Issues in the Semantic Web, 2004.

[15]. Leo Sauermann, DFKI GmbH, and Sebastian Trüg, Mandriva Linux, Germany May 2008, Case Study: KDE 4.0 Semantic Desktop Search and Tagging 